\title{
Rapid changes of sunspot structure associated with solar eruptions
}

\author{
Haimin Wang and Chang Liu \\ Space Weather Research Lab, New Jersey Institute of Technology, Newark, NJ 07102, USA \\ email: haimin.wang@njit.edu
}

\begin{abstract}
In this paper we summarize the studies of flare-related changes of photospheric magnetic fields. When vector magnetograms are available, we always find an increase of transverse field at the polarity inversion line (PIL). We also discuss 1 minute cadence line-of-sight MDI magnetogram observations, which usually show prominent changes of magnetic flux contained in the flaring $\delta$ spot region. The observed limb-ward flux increases while disk-ward flux decreases rapidly and irreversibly after flares. These observations provides evidences, either direct or indirect, for the theory and prediction of Hudson, Fisher \& Welsch (2008) that the photospheric magnetic fields would respond to coronal field restructuring and turn to a more horizontal state near the PIL after eruptions. From the white-light observations, we find that at flaring PIL, the structure becomes darker after an eruption, while the peripheral penumbrae decay. Using highresolution Hinode data, we find evidence that only dark fibrils in the "uncombed" penumbral structure disappear while the bright grains evolve to G-band bright points after flares.
\end{abstract}

Keywords. Sun: activity, sun: flares, sun: coronal mass ejections (CMEs), sun: magnetic fields

\section{Introduction}

Solar eruptions, including flares, filament eruptions, and coronal mass ejections (CMEs) have been understood as the result of magnetic reconnection in the solar corona (e.g., Kopp \& Pneuman 1976; Antiochos et al. 1999). Although surface magnetic field evolution (such as new flux emergence and shear motion) play important roles in building energy and triggering eruption, most models of flares and CMEs have the implication that photospheric magnetic fields do not have rapid, irreversible changes associated with the eruptions. The key reason behind this assumption is that the solar surface, where the coronal magnetic fields are anchored, has much higher density and gas pressure than the corona. Recently, we note the work by Hudson, Fisher \& Welsch (2008, hereafter HFW08), who quantitatively assessed the back reaction on the solar surface and interior resulting from the coronal field evolution required to release energy, and made the prediction that after flares, the photospheric magnetic fields become more horizontal. This is one of the very few models that specifically predict that flares can be accompanied by rapid and irreversible changes of photospheric magnetic fields. Fisher et al. (2010) elaborated this work further.

On the observational side, earlier studies were inconclusive on the flare-related changes of photospheric magnetic field topology. Wang (1992) and Wang et al. (1994) showed impulsive changes of vector fields after flares including some unexpected patterns such as increase of magnetic shear along the PIL, while mixed results were also reported (e.g., Hagyard et al. 1999; Chen et al. 1994). It is not until recently that rapid and permanent changes of photospheric magnetic fields, mainly the line-of-sight component, are observed 

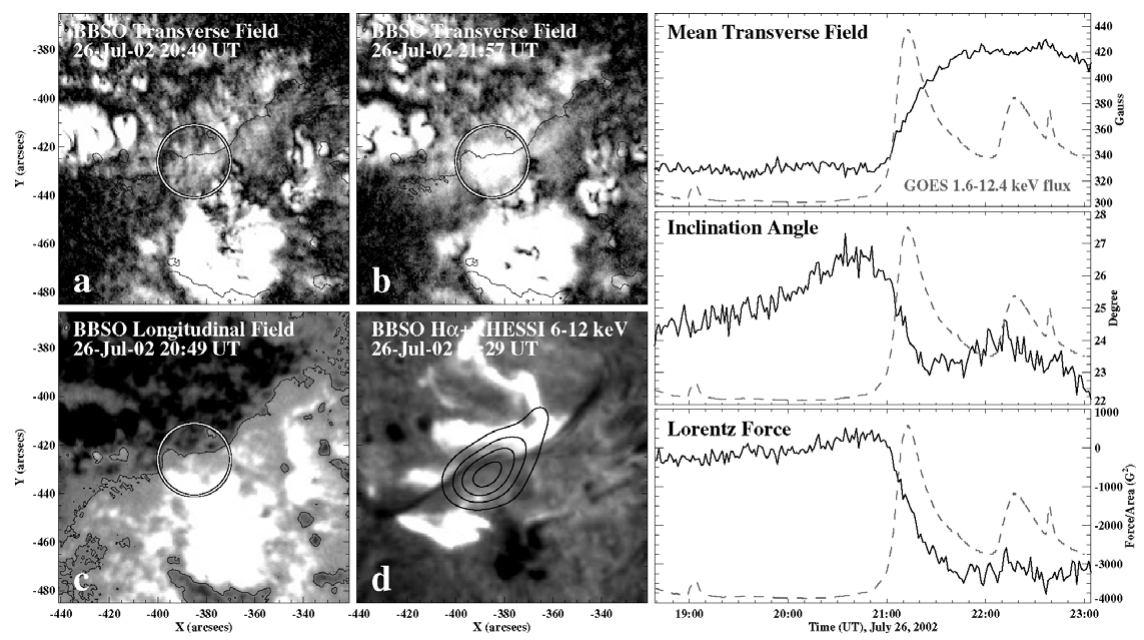

Figure 1. Time profiles of transverse field, inclination angle, and Lorentz force per unit area within a white circled region (in $a-c$ ) at the PIL (black line in $a-c$ ) for the 2002 July 26 M8.7 flare (see an $\mathrm{H} \alpha$ image in $d$ overplotted with RHESSI soft X-ray source), calculated using BBSO vector magnetograms. In GOES 10 soft X-ray flux (dashed line), the flare started at 20:51 UT, peaked at 21:12 UT, and ended at 21:29 UT.

to consistently appear in major flares and considered as indicative of flare energy release (Kosovichev \& Zharkova 2001; Sudol \& Harvey 2005). In particular, a number of papers of our group have been devoted to the finding of sudden unbalanced magnetic flux change (Spirock et al. 2002; Wang et al. 2002; Yurchyshyn et al. 2004; Wang et al. 2004a; Wang 2006) and a new phenomenon of sunspot white-light structure change (Wang et al. 2004b; Wang et al. 2005; Liu et al. 2005; Deng et al. 2005; Chen et al. 2007; Jing et al. 2008; Li et al. 2010) associated with flares. Liu et al. (2005) proposed a reconnection picture where the active region field collapses inward after flares as signified by HFW08.

Wang \& Liu (2010) recently examined the observations in a systematic fashion and compare them quantitatively with the prediction of HFW08. Meanwhile, several conflicting concepts in our earlier papers can be reconciled with new physical understanding.

\section{Observations and Results}

The most straightforward way to determine changes of vector fields associated with flares is to monitor the time sequence of vector magnetograms. In order to detect any rapid and subtle variation, however, magnetogram observations with high cadence (a few minutes), high resolution $\left(1^{\prime \prime}\right)$, and high polarization accuracy are required, and it is understood that $H M I / S D O$ will be able to provide unprecedented data with these characteristics. Yet in the past two decades there are some vector magnetograms available that can tackle this topic with certain limitations. All the results obtained thus far point to the conclusion that transverse magnetic field strength increases at the PIL after major flares (namely, the fields there turn more horizontal), which provides direct and strong observational support for the theory of HFW08. Following HFW08, we further quantify the change of Lorentz force per unit area in the vertical direction using the formula:

$$
\delta f_{z}=\left(B_{z} \delta B_{z}-B_{x} \delta B_{x}-B_{y} \delta B_{y}\right) / 4 \pi
$$




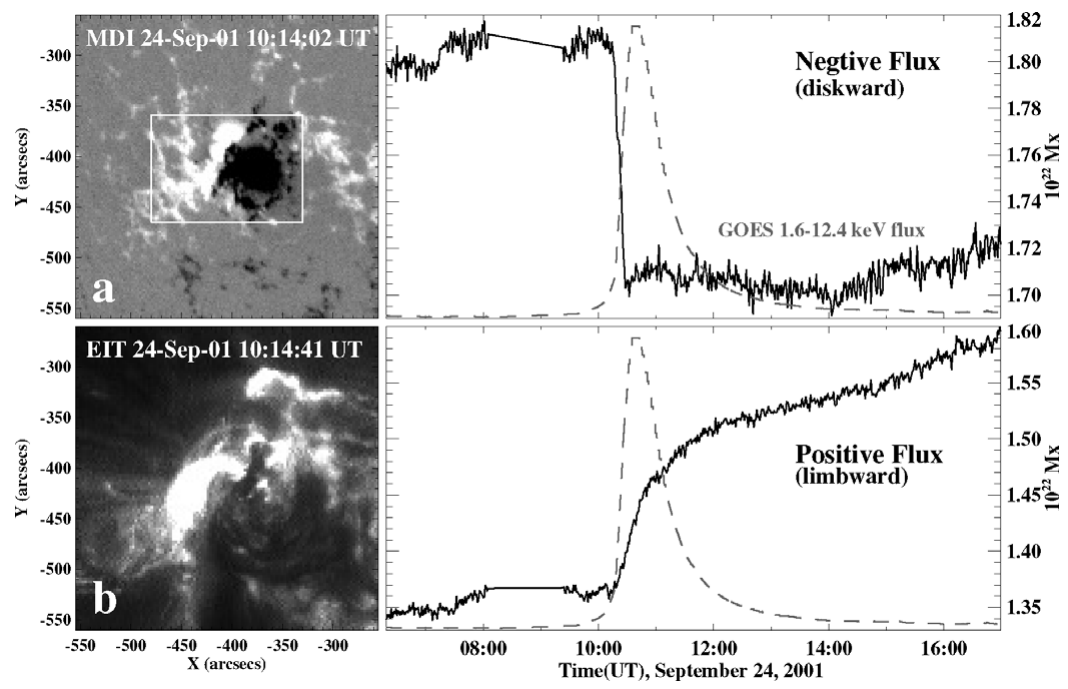

Figure 2. Time profiles of negative and positive MDI line-of-sight magnetic fields within a boxed region (in $a$ ) covering the entire $\delta$ spot for the 2001 September 24 X2.6 flare, seen in an EIT image $(b)$. The flare started at 09:32 UT, peaked at 10:38 UT, and ended at 11:09 UT.

and present an example of our re-analysis of the vector field observations associated with the 2002 July 26 M8.7 flare in Figure 1. For the compact region at the flaring PIL, the results unambiguously show the following. First, the mean transverse field strength increases $90 \mathrm{G}$ in about one hour ensuing from the rapid rising of the flare soft X-ray emission at 20:51 UT. Second, the inclination angle decreases $3^{\circ}$ accordingly, which indicates that magnetic field lines there turn to a more horizontal direction as predicted by HFW08. Third, the change of the Lorentz force per unit area is $\sim-5000 \mathrm{G}^{2}$, integrating which over the analyzed area of $\sim 3.2 \times 10^{18} \mathrm{~cm}^{2}$ yields a downward net Lorentz force in the order of $1.6 \times 10^{22}$ dynes, comparable to what is expected by HFW08. Similar results have been found for other flares as listed in Table 1 of Wang \& Liu (2010).

Considering that vector magnetograms covering major flares with sufficient cadence and quality are rare and that reliable detection of rapid changes in the observed magnetic signals would ideally require stable observing conditions, we use the line-of-sight magnetograms $\left(\sim 2^{\prime \prime}\right.$ pixel $\left.^{-1}\right)$ measured with $S O H O /$ MDI to study more events, taking advantage of its long-sequence and seeing-free data set. We surveyed all the X-class flares satisfying our event selection criteria, and analyzed 18 events. Except for one case, all events exhibit an increase of limbward flux and a decrease of diskward flux of active region magnetic fields after flares with an order of magnitude of $10^{20} \mathrm{Mx}$ (no obvious changes can be detected for one of the two polarities in some flares). Figure 2 shows the field changes associated with the 2001 September 24 X2.6 flare at NOAA AR 09632, which are calculated for the entire $\delta$ spot region. It can be clearly seen that the limbward/diskward (positive/negative) fluxes increase/decrease for a similar amount right after the flare impulsive phase. The change-over time for positive and negative fluxes are 40 and 10 minutes, respectively. As a matter of fact, these behaviors of line-of-sight fields also imply that the active region magnetic fields become more horizontal after flares, which was indicated by Wang (2006) based on limited sample events. 


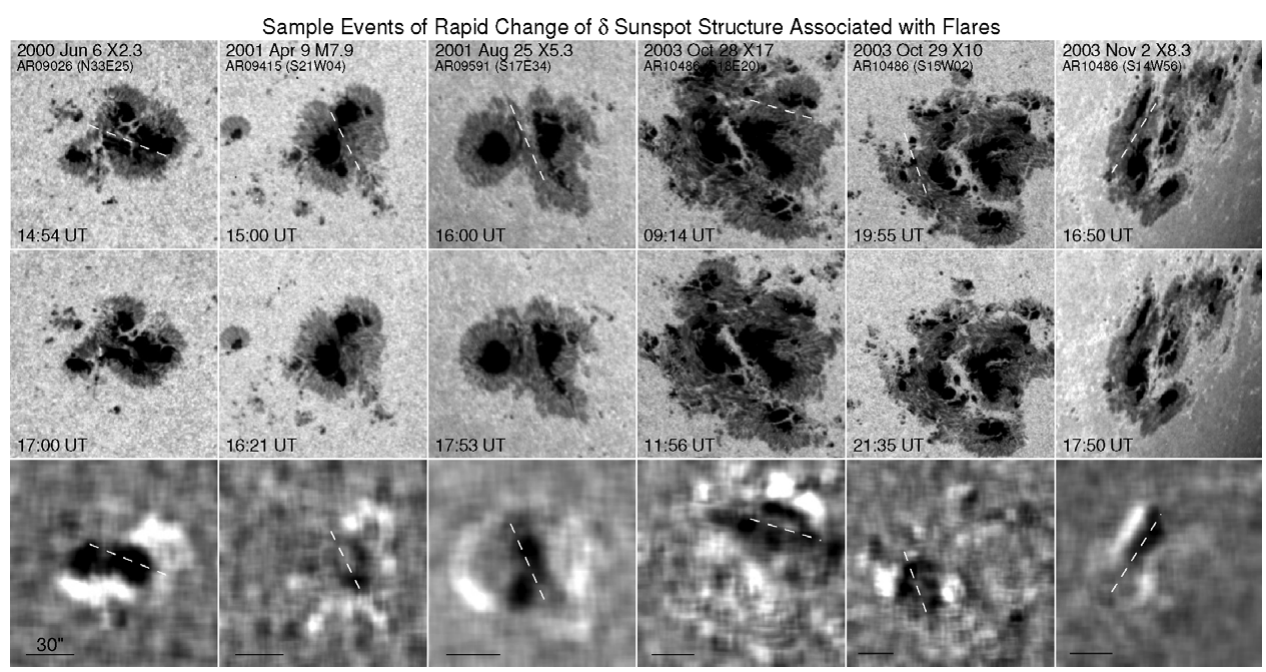

Figure 3. TRACE white-light images revealing the rapid change of $\delta$ sunspot structure associated with six major flares. The top, middle, and bottom rows show the pre-flare images, post-flare images, and the difference images between them, respectively. The white feature in the difference image indicates the region of penumbral decay, while the dark feature indicates the region of central umbral/penumbral enhancement. For each event, the white dashed line denotes the approximate flaring PIL and the black line represents a scale of $30^{\prime \prime}$ (adapted from Liu et al. 2005).

From more recent investigation, we began to appreciate the consistent pattern of magnetic field changes associated with flares using simple white-light observations (Wang et al. 2004b; Liu et al. 2005; Deng et al. 2005). The most outstanding changes are the decay of penumbral structure in the outer sides of the $\delta$ spot and the enhancement of sunspot structure near the flaring PILs. Figure 3 demonstrates examples of sunspot structure change. The difference between post- and pre-flare images always shows a dark patch at the flaring magnetic polarity inversion line surrounded by a bright ring, corresponding to the enhancement of center sunspot structure and decay of peripheral penumbra. These examples were discussed in detail by Liu et al. (2005), where we showed that these rapid changes were associated with the flares and were irreversible.

It is recently found that the sunspot penumbra has two components forming an "uncombed" structure, i.e., a more inclined dark component added to the bright grains that have a more vertical field structure (e.g., Langhans et al., 2005). Therefore, it is natural to ask how do the two components evolve in the above-mentioned penumbral decay. We investigated the X6.5 flare on 2006 December 6 and show the evolution of the G-band images in Figure 4. The penumbral decay is obvious in the region marked by a white eclipse. Examining the time-lapse movie, it is evident that the penumbral decay may make the dark fibrils in the penumbrae disappear, while the bright grains evolve to the G-band bright points corresponding to vertical magnetic flux tubes.

\section{Summary and Discussion}

Synthesizing the research of flare-related rapid and irreversible changes in both vector and line-of-sight magnetic fields, we have revealed a pattern that magnetic fields near the PIL become more horizontal, while peripheral penumbral fields become more vertical. 


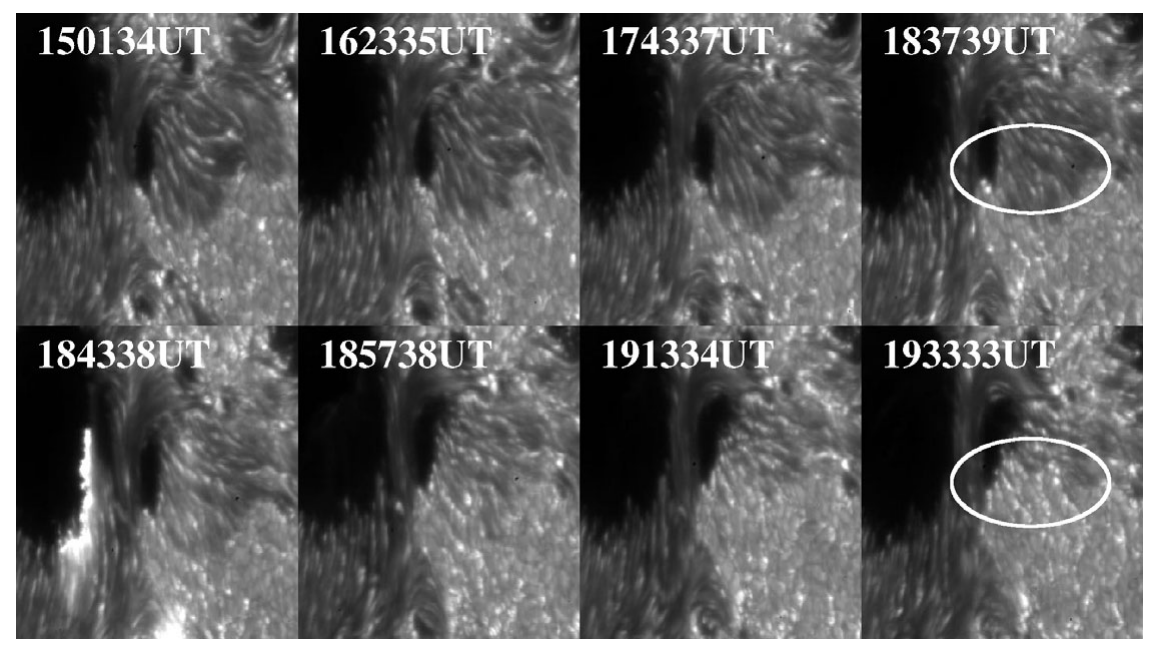

Figure 4. Time sequence of G-band images as observed by Hinode/SOT covering the X6.5 flare on 2006 December 6 . The white eclipse marks the most prominent region of penumbral decay. The while-light flare is evident in the frame at 18:43:38 UT. The field of view is $43^{\prime \prime} \times 48^{\prime \prime}$.

The change-over time lies between $\sim 10$ minutes to 1 hour, and all the changes are cotemporal with the flare initiation. We discussed the results in the context of HFW08. However, as the result of the discussion in this Symposium, the fields near the PIL to become compressed after flare/CME may be due to relaxation of the magnetic stresses (Klimchuk, 1990). The Figure 2 in that paper may also explain the observed penumbral decay.

\section{References}

Antiochos, S. K., DeVore, C. R., \& Klimchuk, J. A., 1999, ApJ, 510, 485

Chen, J., Wang, H., Zirin, H., \& Ai, G., 1994, Solar Phys., 154, 261

Chen, W., Liu, C., \& Wang, H., 2007, ChJAA, 7, 733

Deng, N., Liu, C., Yang, G., \& Wang, H., Denker C., 2005, ApJ, 623, 1195

Fisher, G. H., Bercik, D. J., Welsch, B. T., \& Hudson, H. S., 2010, arXiv:1006.5247

Hagyard et al., 1999, Solar Phys., 184, 133

Hudson, H. S., Fisher, G. H., \& Welsch, B. T., 2008, ASP Conference Series, 383, 221

Jing, J., Wiegelmann, T., Suematsu, Y., Kubo, M., \& Wang, H., 2008, ApJL, 676, L81

Klimchuck, J. A., 1990, ApJ, 354, 745

Kopp, R. A. \& Pneuman, G. W., 1976, Solar Phys., 50, 85

Kosovichev, A. G. \& Zharkova, V. V. 2001, ApJL, 550, L105

Langhans, K., Scharmer, G. B., Kiseman, D., Lofdahl, M. G., \& Berger, T. E., 2005, A $\mathcal{G} A$, 436,1087

Li, Y., Jing, J., Fan, Y., \& Wang, H., 2010, this proceeding

Liu, C., Deng, N., Liu, Y., Falconer, D., Goode, P. R., Denker, C., \& Wang, H., 2005, ApJ, 622, 722

Spirock, T. J., Yurchyshyn, V. B., \& Wang, H., 2002, ApJ, 572, 1072

Sudol, J. J. \& Harvey, J. W., 2005, ApJ, 635, 647

Wang, H., 1992, Solar Phys., 140, 85

Wang, H., 2006, ApJ, 649, 490

Wang, H. \& Liu, C., 2010, ApJL, 716, L195

Wang, H., Ewell, M. W., Zirin, H., \& Ai, G. 1994, ApJ, 424, 436

Wang, H., Liu, C., Qiu, J., Deng, N., Goode, P. R., \& Denker, C., 2004b, ApJL, 601, L195 
Wang, H., Liu, C., Zhang, H., \& Deng, Y, 2005, ApJ, 627, 1031

Wang, H., Qiu, J., Jing, J., Spirock, T. J., \& Yurchyshyn, V., 2004a, ApJ, 605, 931

Wang, H., Spirock, T. J., Qiu, J., Ji, H., Yurchyshyn, V., Moon, Y. J., Denker, C., \& Goode, P. R., 2002, ApJ, 576, 497

Yurchyshyn, V. B., Wang, H., Abramenko, V., Spirock, T. J., \& Krucker, S., 2004, ApJ, 605, 546 